\title{
Adaptive Relaying Protocol for Wireless Power Transfer and Information Processing
}

DOI:

10.1109/LCOMM.2016.2593877

\section{Document Version}

Accepted author manuscript

Link to publication record in Manchester Research Explorer

\section{Citation for published version (APA):}

Tao, R., Salem, A., \& Hamdi, K. (2016). Adaptive Relaying Protocol for Wireless Power Transfer and Information Processing. IEEE Communications Letters, 20(10), 2027-2030. https://doi.org/10.1109/LCOMM.2016.2593877

\section{Published in:}

IEEE Communications Letters

\section{Citing this paper}

Please note that where the full-text provided on Manchester Research Explorer is the Author Accepted Manuscript or Proof version this may differ from the final Published version. If citing, it is advised that you check and use the publisher's definitive version.

\section{General rights}

Copyright and moral rights for the publications made accessible in the Research Explorer are retained by the authors and/or other copyright owners and it is a condition of accessing publications that users recognise and abide by the legal requirements associated with these rights.

\section{Takedown policy}

If you believe that this document breaches copyright please refer to the University of Manchester's Takedown Procedures [http://man.ac.uk/04Y6Bo] or contact uml.scholarlycommunications@manchester.ac.uk providing relevant details, so we can investigate your claim.

\section{OPEN ACCESS}




\title{
Adaptive Relaying Protocol for Wireless Power Transfer and Information Processing
}

\author{
Ran Tao, Abdelhamid Salem, Student Member, IEEE and Khairi Ashour Hamdi, Senior Member, IEEE.
}

\begin{abstract}
In this paper, an amplify-and-forward (AF) relaying system is considered, where an energy constrained relay node harvests energy from the received radio frequency (RF) signal and uses this harvested energy to amplify and forward the source signal to the destination. Based on the time switching (TS) and power splitting (PS) receiver architectures, an adaptive receiving architecture for energy harvesting and information processing is proposed and an adaptive relaying (AR) protocol based on it is developed to enable energy harvesting and information processing at the relay. In light of this, analytical expressions of throughput are derived for both delaylimited transmission (DLT) and delay-tolerant transmission (DTT) modes, when the AR protocol is implemented at the relay. Monte Carlo simulations are provided throughout to validate our analysis and the impact of some important system parameters on the adopted performance metric are investigated. In addition, we compare the system performance in terms of the throughput of AR protocol with PS relaying and TS relaying protocols proposed in [1]. Results show that, the AR protocol has a better system performance around the point in which there is a throughput crossover for both PS relaying and TS relaying protocols.
\end{abstract}

Index Terms-Energy harvesting, cooperative communications, wireless power transfer, relaying protocol.

\section{INTRODUCTION}

$\mathbf{E}$ NERGY harvesting $(\mathrm{EH})$ has recently attracted considerable attention in wireless communication field. This technology becomes a solution of inaccessible battery-limited devices to prolong the life cycle. This idea comes from the fact that RF signals can transfer information and power at the same time, therefore, it allows nodes to harvest power and process information concurrently, which is called simultaneous wireless information and power transfer (SWIPT) [2]-[5]. This concept was developed in [2], where a tradeoff between $\mathrm{EH}$ and information transmission was considered. This work was extended to frequency-selective channels in [3]. However, these studies focused on ideal receivers, which means that $\mathrm{EH}$ and information processing can be achieved simultaneously, this assumption seems unrealistic in practice. For more practical, two practical EH receivers, time switching (TS) and power splitting (PS) were proposed in [4] and [6]. In TS the whole period is divided into two parts, used for $\mathrm{EH}$ and information processing, whereas in the PS scheme, the signal is divided into two fractions, aiming at EH and information processing, respectively. The authors in [1] investigated the throughput of energy-constrained amplifyand-forward (AF) relaying system, in which the relay solely relies on EH from the received information signal. Moreover, the authors proposed two relaying protocols, time switching relaying (TSR) and power splitting relaying (PSR). In [7] the authors combined the antenna selection and PS techniques in AF relaying systems. In [8] the concept of partial network level cooperation for $\mathrm{EH}$ networks was introduced. The authors in [9] studied the impact of energy constraints on a relay-aided wireless network in which

The authors are with the School of Electrical and Electronic Engineering, the University of Manchester, Manchester, M13 9PL, UK. (emails: sherrytaoran@163.com and \{abdelhamid.salem, k.hamdi\}@ manchester.ac.uk). the source and relay nodes both have EH capabilities and unlimited battery capacity. In [10] wireless $\mathrm{EH}$ and information transfer in cognitive relay networks was studied. The two traditional TS and PS architectures have been widely studied in literature, many of these studies have compared the performance of the two EH receivers under different scenarios, for instance [11]-[14]. From these studies, it is found that, the PSR and TSR protocols have some drawbacks, for instance TSR has to loss some information while it switches to the harvesting mode and PSR has a low coverage area.

In this paper, based on the TS and PS receivers, an adaptive receiver architecture (ARA) for $\mathrm{EH}$ and information processing is proposed. In the ARA, the receiver spends a fraction of time for $\mathrm{EH}$ and the remaining time for information processing; it works here as a TS receiver. After that, a fraction of the received signal power is also used for $\mathrm{EH}$ and the remaining power for information processing; it works here as a PS receiver, as shown in Fig. 1. Therefore, this new architecture combined the two traditional EH receivers TS and PS in one adaptive receiver. Then we implement ARA at a relay node in three nodes cooperative system and adaptive relaying (AR) protocol is developed, AR protocol will be explained in details in the next section. Based on AR protocol, we derive analytical expressions for the throughput in delay limited transmission (DLT) and in delay tolerant transmission (DTT) modes. In DLT, the destination can decode the received signal block by block and the code length should not be longer than the the transmission block time. Consequently, the source transmits data at fixed rate and the average throughput is obtained by calculating the outage probability. In DTT, the destination can store the received blocks in a buffer and then can tolerate the delay for decoding the received signals. Consequently, the code length can be longer than transmission block time and the source transmits data at rate less than or equal to the ergodic capacity. The average throughput in DTT is obtained by evaluating the ergodic capacity. The results reveal that, the AR protocol works as PSR when PSR is the best and as TSR when TSR is the best. In addition, the AR protocol outperforms PSR and TSR around the point in which there is a throughput crossover for both PSR and TSR protocols. Therefore, the main contributions of this paper are summarized as follows: 1) New ARA for EH and information processing is proposed 2) New AR protocol to enable SWIPT at the energy constrained relay node is developed. 3) analytical expressions for throughput are derived 4) in order to investigate the effectiveness of AR protocol, the performance of the AR protocol is compared with TSR and PSR protocols.

\section{SyStem MODEL}

We consider the system model shown in Fig. 2, where the source transmits RF information signal to the destination node through an energy constrained relay node; all the nodes are equipped with a single antenna. The channels and the distances between the nodes are donated as $h, g$ and $d_{1}, d_{2}$, respectively, as in Fig. 2. We assume that, AF relaying protocol is adopted and all the channels in this 


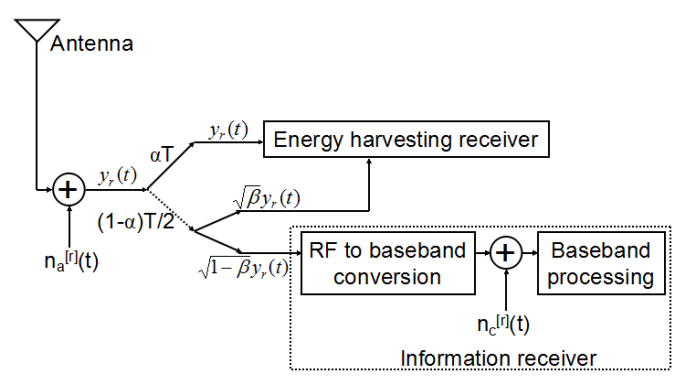

Figure 1: Block diagram of ARA.

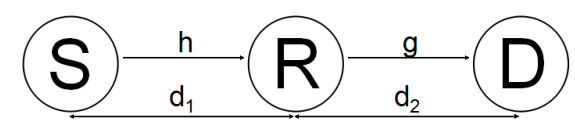

Figure 2: System model for energy constrained relay system.

model are quasi-static block fading channels, following Rayleigh distributed magnitude. Due to the deep shadowing, there is no direct link between the source and the destination. The impact of the direct link will be presented in section IV. In addition, the source and destination both have unlimited power supply, while the relay solely relies on the harvested energy from RF signals based on AR protocol. The communication between the source and the destination achieves over two phases. In phase I, the source transmits signal to the relay, the relay processes information and harvests energy by using AR protocol. In phase II, the relay uses the harvested energy to amplify and forward the information signal to the destination. Fig 3 illustrates the key parameters in the AR protocol, where $T$ is the block time, in which the source transmits information block to the destination, $\alpha$ is a fraction of $T$ used by the relay to harvest energy, $0<\alpha<1$, and $\beta P$ is a fraction of received signal power, $0<\beta<1$. In the AR protocol scheme, during a fraction of time $\alpha T$, the relay harvests energy from the received signal. Half of the remaining time $(1-\alpha T) / 2$ is allocated for source to relay transmission and $(1-\alpha T) / 2$ for relay to destination transmission (like the TSR protocol). Then, a fraction of the received power $\beta P$ is also harvested by the relay and the other part $(1-\beta) P$ is used for information processing (like the PSR protocol), as shown in Fig. 3 . The received signal at the relay in phase I is given by

$$
y_{r}(t)=\frac{1}{\sqrt{d_{1}^{m}}} \sqrt{P_{s}} h s(t)+n_{a[r]}(t)
$$

where $P_{s}$ is the transmitted source power, $m$ is the path loss exponent, $s(t)$ is the normalized source signal, $E\left\{|s(t)|^{2}\right\}=1$ and $n_{a[r]}(t)$ is the additive white Gaussian noise (AWGN) introduced by the receiving antenna at the relay with variance $\sigma_{n a[r]}^{2}$. Therefore, the amount of harvested energy can be given by

$$
E_{h}=\frac{\eta_{\alpha} P_{s}|h|^{2} \alpha T}{d_{1}^{m}}+\frac{\eta_{\beta} \beta P_{s}|h|^{2}(1-\alpha) T}{2 d_{1}^{m}}
$$

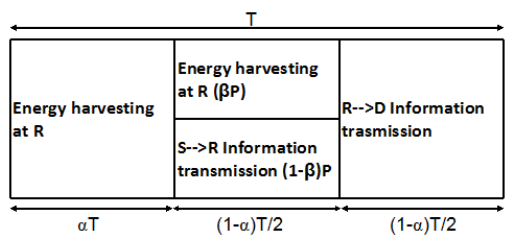

Figure 3: Key parameters of AR protocol. where $\eta_{\alpha}$ and $\eta_{\beta}$ represent the EH efficiency of the two stages, TS and PS, respectively, $0<\eta_{\alpha}<1$ and $0<\eta_{\beta}<1$. Now, the transmitted power from the relay can be given by, $P_{r}=\frac{E_{h}}{(1-\alpha) T / 2}$. After the conversion to base-band as in Fig. 1, the base band signal at the relay can be expressed by

$$
y_{r}(k)=\frac{\sqrt{(1-\beta) P_{s}}}{\sqrt{d_{1}^{m}}} h s(k)+\sqrt{(1-\beta)} n_{a[r]}(k)+n_{c[r]}(k)
$$

where $k$ is the symbol index, $n_{c[r]}(k)$ is the AWGN due to the signal conversion, with variance $\sigma_{n c[r]}^{2}, s(k)$ is the sampled information signal. After that, the relay transmits the following signal

$$
x_{r}(k)=\sqrt{\frac{P_{r}}{F}} y_{r}(k)
$$

where $F$ is the power constraint factor, which is given by, $F=$ $\frac{(1-\beta) P_{s}|h|^{2}}{d_{1}^{m}}+(1-\beta) \sigma_{n a[r]}^{2}+\sigma_{n c[r]}^{2}$. The received signal at the destination can be written as

$$
y_{d}(k)=\frac{g}{\sqrt{d_{2}^{m}}} x_{r}(k)+n_{[d]}(k)
$$

where $n_{[d]}(k)$ is the AWGN at the destination with variance $\sigma_{n d}^{2}$. Substituting (4) into (5) the received destination signal can be given as in (6), shown at the top of the next page. Consequently, the signalto-noise ratio (SNR) at the destination node, $\gamma_{d}$, can be written as

$$
\gamma_{d}=\frac{\varepsilon_{1}|h|^{4}|g|^{2}}{\varepsilon_{2}|h|^{2}|g|^{2}+\varepsilon_{3}|h|^{2}+\varepsilon_{4}}
$$

where $\varepsilon_{1}, \varepsilon_{2}, \varepsilon_{3}, \varepsilon_{4}$ are $\varepsilon_{1}=2 \eta_{a} P_{s}(1-\beta) \alpha+\eta_{b} \beta P_{s}(1-$ $\beta)(1-\alpha), \varepsilon_{2}=2 \eta_{a} P_{s} \alpha d_{1}^{m}(1-\beta) \sigma_{n a[r]}^{2}+\eta_{b} \beta P_{s}(1-\alpha) d_{1}^{m}(1-$ $\beta) \sigma_{n a[r]}^{2}+2 \eta_{a} P_{s} \alpha d_{1}^{m} \sigma_{n c[r]}^{2}+\eta_{b} \beta P_{s}(1-\alpha) d_{1}^{m} \sigma_{n c[r]}^{2}, \varepsilon_{3}=(1-$ 及) $P_{s} \sigma_{n d}^{2} d_{1}^{m} d_{2}^{m}(1-\alpha)$ and $\varepsilon_{4}=(1-\beta) P_{s} \sigma_{n d}^{2} d_{1}^{m} d_{2}^{m}(1-\alpha)+$ $\sigma_{n c[r]}^{2} d_{1}^{2 m} d_{2}^{m} \sigma_{n d}^{2}(1-\alpha)$.

\section{Throughrut ANALYSiS}

In this section, the throughput $(\tau)$ for both DLT and DTT modes are analyzed as follow.

\section{A. Delay-Limited Transmission DLT}

In the DLT mode, $\tau$ is derived by calculating the outage probability, $p_{\text {out }}$, at a fixed source transmission rate $(R), p_{\text {out }}$ can be expressed as

$$
p_{\text {out }}=p\left\{\gamma_{d}<\gamma_{0}\right\}
$$

where $\gamma_{0}$ is the threshold value of SNR, $\gamma_{0}=2^{R}-1$ and $R \triangleq$ $\log _{2}\left(1+\gamma_{0}\right)$. Substituting (7) into (8) we can get

$$
p_{\text {out }}=p\left\{\left(A|h|^{4}-B|h|^{2}\right)|g|^{2}<C|h|^{2}+D\right\}
$$

where $A, B, C, D$ are $A=\varepsilon_{1}, B=\gamma_{0} \varepsilon_{2}, C=\gamma_{0} \varepsilon_{3}$ and $D=\gamma_{0} \varepsilon_{4}$. From (9) we can write $p_{\text {out }}$ as [1]

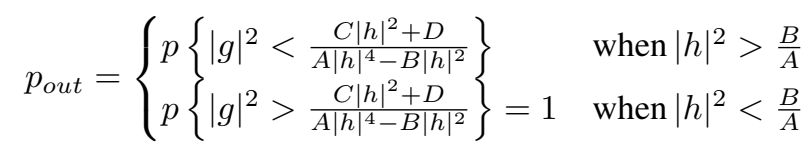

The second probability in (10) can be explained by the fact that, if $|h|^{2}<\frac{B}{A}, A|h|^{4}-B|h|^{2}$ will be negative and $|g|^{2}$ is always positive. Now, we can write $p_{\text {out }}$ as 


$$
y_{d}(k)=\sqrt{\frac{P_{r}}{F d_{2}^{m}}} g\left(\frac{\sqrt{(1-\beta) P_{s}}}{\sqrt{d_{1}^{m}}} h s(k)+\sqrt{(1-\beta)} n_{a[r]}(k)+n_{c[r]}(k)\right)+n_{[d]}(k)
$$

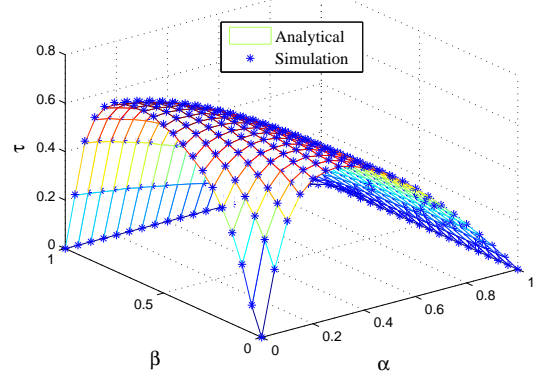

(a) Analytical and simulation throughput in DLT

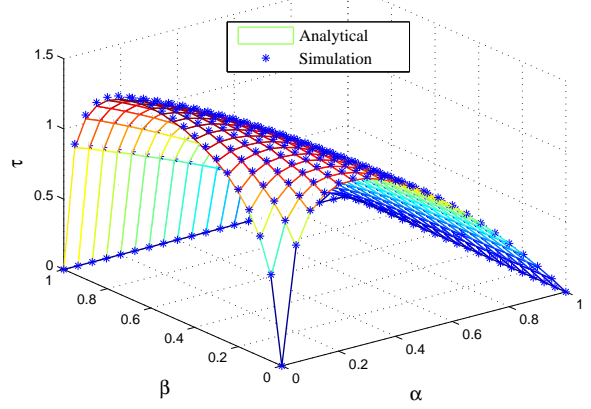

(b) Analytical and simulation throughput in DTT

Figure 4: The throughput of DLT and DTT transmission modes.

$$
p_{\text {out }}=\int_{0}^{B / A} f_{|h|^{2}}(z) d z+\int_{B / A}^{\infty} f_{|h|^{2}}(z) F_{|g|^{2}}(z) d z
$$

The probability density function (PDF) of $|h|^{2}$ and the cumulative density function (CDF) of $|g|^{2}$ are given by, respectively, $f_{|h|^{2}}(z)=$ $\frac{1}{\lambda^{h}} e^{-\frac{z}{\lambda_{h}}}$ and $F_{|g|^{2}}(z)=1-e^{-\frac{z}{\lambda_{g}}}$, where $\lambda_{h}$ and $\lambda_{g}$ are the mean values. Substituting $f_{|h|^{2}}(z)$ and $F_{|g|^{2}}(z)$ into (11) we can get

$$
p_{\text {out }}=1-\frac{1}{\lambda_{h}} \int_{\frac{B}{A}}^{\infty} e^{-\left[\frac{z}{\lambda_{h}}+\frac{C z+D}{\left(A z^{2}-B z\right) \lambda_{g}}\right]} d z
$$

Finally, the throughput of DLT is $\tau=\frac{\left(1-p_{\text {out }}\right)(1-\alpha) R}{2}$.

\section{B. Delay-Tolerant Transmission DTT}

The throughput in DTT mode is derived by calculating the ergodic capacity $\left(\bar{C}_{d}\right)$, which can be expressed by

$$
\bar{C}_{d}=E\left\{\log _{2}\left(1+\gamma_{d}\right)\right\}=\int_{0}^{\infty} \log _{2}\left(1+\gamma_{d}\right) f_{\gamma_{d}}(\gamma) d \gamma
$$

where $E\{$.$\} is the expectation operation. The PDF of SNR f_{\gamma_{d}}(\gamma)$ can be found by differentiation (12) as

$$
f_{\gamma_{d}}(\gamma)=\frac{1}{\lambda_{h} \gamma} \int_{\frac{B}{A}}^{\infty} \frac{(C z+D) A z^{2}}{\left(A z^{2}-B z\right)^{2} \lambda_{g}} e^{-\left[\frac{z}{\lambda_{h}}+\frac{C z+D}{\left(A z^{2}-B z\right) \lambda_{g}}\right]} d z
$$

Substituting (14) into (13), we can get $\bar{C}_{d}$ as in (15), shown at the top of the next page. Finally, the throughput in DTT is given by $\tau=\frac{(1-\alpha) \overline{C_{d}}}{2}$.

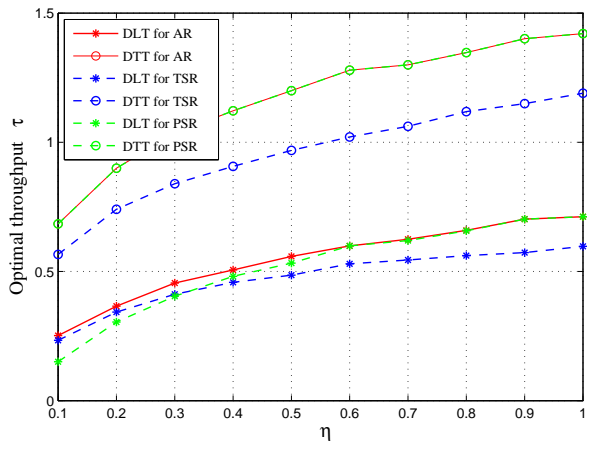

Figure 5: Effect of EH efficiency on the system performance.

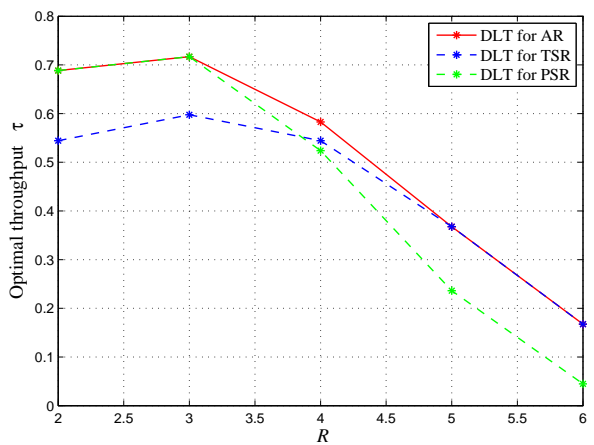

Figure 6: Effect of transmission rate on the system performance.

\section{Numerical Results}

In this section we present numerical results for the expressions derived above. Monte Carlo simulations with $10^{6}$ independent trials are conducted in which channel coefficients are randomly generated in each simulation run. Unless it is mentioned, the transmission rate at the source is chosen to be $3 \mathrm{bits} / \mathrm{sec} / \mathrm{Hz}$, EH efficiencies $\eta_{\alpha}$ and $\eta_{\beta}$ are set to 1 , source power $P_{s}=1 \mathrm{~W}$, and the path loss exponent is 2.7 , the distances $d_{1}$ and $d_{2}$ are normalized to unit value. For simplicity, similar noise variances at the nodes are assumed $\sigma^{2}=0.01 \mathrm{~W}$, and $\lambda_{h}$ and $\lambda_{g}$ are set to 1 .

\section{A. Effect of EH Time and PS Factors}

Fig. 4 shows the throughput with respect to $\beta$ and $\alpha$ for DLT and DTT modes. From this figure it is clear that, the throughput increases as $\beta$ and $\alpha$ increase to optimal values, but later it starts decreasing as $\beta$ and $\alpha$ increase from its optimal values. In this case the AR works exactly as PSR which provides optimal performance.

\section{B. Effect of EH Efficiency and Transmission Rate}

In this sub-section we consider the impact of the EH efficiency and transmission rate on the system performance. Fig. 5 plots the throughput versus EH efficiency, for simplicity we assume $\eta=\eta_{\alpha}=$ $\eta_{\beta}$. From this figure we can see in DTT mode PSR always has a better system performance, so the AR in DTT mode always works as PSR. However, in the DLT mode, when $\eta$ is low, longer time is required to harvest sufficient energy, in this case the AR outperforms both PSR and TSR in the range from $\eta=0.1$ to 0.6 , i.e., when the AR is in the transition state from TSR to PSR. In addition, in 


$$
\bar{C}_{d}=\frac{1}{\lambda_{g} \lambda_{h}} \int_{0}^{\infty} \int_{\frac{B}{A}}^{\infty} \log _{2}(1+\gamma) \frac{(C z+D) C z^{2}}{\left(A z^{2}-B z\right)^{2} \gamma} e^{-\left[\frac{z}{\lambda_{h}}+\frac{C z+D}{\left(A z^{2}-B z\right) \lambda_{g}}\right]} d z d \gamma
$$

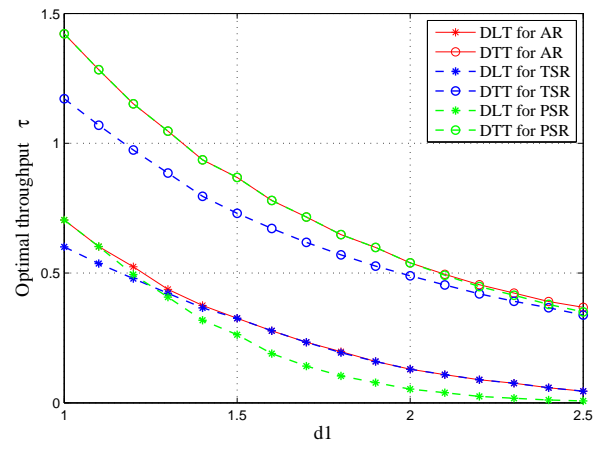

(a) Effect of distance $d_{1}$.

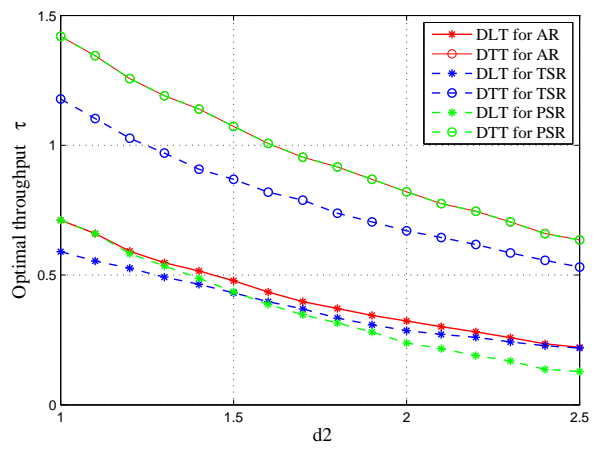

(b) Effect of distance $d_{2}$.

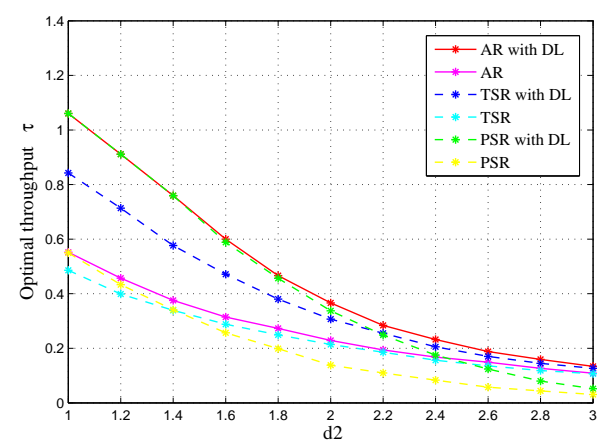

(c) Effect of DL on the system performance.

Figure 7: Effect of distances and the DL on the system performance.

order to examine the impact of the source transmission rate $R$ on the system performance, we vary $R$ from 2 to 6 as shown in Fig. 6. As we can see from this figure as $R$ enhances the throughput firstly increases as expected and the AR in this period works exactly as PSR, after that the throughput starts decreasing as $R$ increases further. In high transmission rate, the AR works exactly as TSR, while in the transition state from 3 to $6 \mathrm{bit} / \mathrm{sec} / \mathrm{Hz}$, the AR has a higher throughput than PSR and TSR.

\section{Effect of Distances and the Direct Link}

In order to investigate the impact of $d_{1}$ and $d_{2}$ on the throughput, we fixed one of them, while the other one changes from 1 to $2.5 \mathrm{~m}$. Fig. 7 shows the throughput versus $d_{1}$ and $d_{2}$, respectively. From the figure we can observe that, the AR turns to have better performance than PSR and TSR along with the distance increases from normalized one. This superiority can be observed in DLT mode when the AR is in the transition state from PSR to TSR, in Fig. 7a in the period from $d_{1}=1.2 \mathrm{~m}$ to $1.36 \mathrm{~m}$ the $\mathrm{AR}$ is in the transition state, while in Fig $7 \mathrm{~b}$ the transition state is in the period from $d_{2}=1.2 \mathrm{~m}$ to $d_{2}=2.5 \mathrm{~m}$. This can be justified by the fact that, when the distance is not long, the relay requires little energy. Therefore, the AR works exactly as PSR, and there is no information missing. However, as the distance increases, the PSR becomes unable to harvest the required power. In this case, the AR allocates another time fraction to harvest energy, and as a consequence the AR has larger coverage area than PSR. It can be concluded from Fig. 7 that, increasing $d_{1}$ has more negative impacts on the throughput than that of $d_{2}$, and the advantage of AR becomes more obvious when $d_{2}$ is larger than 1 . Therefore, the optimal relay location is closer to the source. In order to consider the impact of the direct link (DL), we assume the destination can receive the source signal in the two phases and the destination performs maximum ratio combination (MRC), in order to maximize the received SNR. In this case, we present the throughput versus different values of $d_{2}$ in DLT mode. In Fig. $7 \mathrm{c}$ as we can see, adding the DL can make the superiority of AR less obvious because the impact of $\mathrm{EH}$ on the system performance reduced.

\section{CONCLUSION}

In this paper a wireless cooperative system has been studied, where the relay harvests energy to amplify and forward the source signal to the destination. Based on the proposed ARA, a new relaying protocol, AR protocol, is developed to enable EH and information processing. Analytical expressions for the throughput in DLT and DTT modes are derived. The results reveal that wherever there is a throughput crossover point of both traditional architectures, around this point the AR protocol has a better system performance.

\section{REFERENCES}

[1] A. Nasir, X. Zhou, S. Durrani, and R. Kennedy, "Relaying protocols for wireless energy harvesting and information processing," IEEE Wireless Commun., vol. 12, pp. 3622-3636, Jul. 2013.

[2] L. Varshney, "Transporting information and energy simultaneously," in Proc. IEEE Int. Symp. Inf. Theory (ISIT), pp. 1612-1616, Jul. 2008.

[3] P. Grover and A. Sahai, "Shannon meets tesla: Wireless information and power transfer," in Proc. IEEE Int. Symp. Inf. Theory (ISIT), pp. 2363-2367, Jun. 2010.

[4] X. Zhou, R. Zhang, and C. K. Ho, "Wireless information and power transfer: Architecture design and rate-energy tradeoff," IEEE Trans. Commun., vol. 61, pp. 4754-4767, Nov. 2013.

[5] A. M. Fouladgar and O. Simeone, "On the transfer of information and energy in multi-user systems," IEEE Communications Letters, vol. 16, pp. 1733-1736, November 2012.

[6] R. Zhang and C. K. Ho, "MIMO broadcasting for simultaneous wireless information and power transfer," IEEE Trans. Wireless Commun., vol. 12, pp. 1989-2001, May 2013.

[7] Z. Zhou, M. Peng, Z. Zhao, and Y. Li, "Joint power splitting and antenna selection in energy harvesting relay channels," IEEE Signal Processing Letters, vol. 22, pp. 823-827, July 2015.

[8] M. Kashef and A. Ephremides, "Optimal partial relaying for energy-harvesting wireless networks," IEEE/ACM Transactions on Networking, vol. 24, pp. 113122, Feb 2016.

[9] N. Pappas, M. Kountouris, J. Jeon, A. Ephremides, and A. Traganitis, "Effect of energy harvesting on stable throughput in cooperative relay systems," Journal of Communications and Networks, vol. 18, pp. 261-269, April 2016.

[10] Z. Wang, Z. Chen, B. Xia, L. Luo, and J. Zhou, "Cognitive relay networks with energy harvesting and information transfer: Design, analysis, and optimization," IEEE Transactions on Wireless Communications, vol. 15, pp. 2562-2576, April 2016.

[11] M. Ju, K. M. Kang, K. S. Hwang, and C. Jeong, "Maximum transmission rate of $\mathrm{psr} / \mathrm{tsr}$ protocols in wireless energy harvesting df-based relay networks," IEEE Journal on Selected Areas in Communications, vol. 33, pp. 2701-2717, Dec 2015.

[12] Y. Gu and S. A $\tilde{A}^{-}$ssa, "Rf-based energy harvesting in decode-and-forward relaying systems: Ergodic and outage capacities," IEEE Transactions on Wireless Communications, vol. 14, pp. 6425-6434, Nov 2015.

[13] A. Salem, K. A. Hamdi, and K. M. Rabie, "Physical layer security with rf energy harvesting in af multi-antenna relaying networks," IEEE Transactions on Communications, vol. PP, no. 99, pp. 1-1, 2016.

[14] X. Lu, P. Wang, D. Niyato, D. I. Kim, and Z. Han, "Wireless networks with rf energy harvesting: A contemporary survey," IEEE Communications Surveys Tutorials, vol. 17, pp. 757-789, Secondquarter 2015. 